http://dx.doi.org/10.11646/zootaxa.3646.2.4

http://zoobank.org/urn:1sid:zoobank.org:pub:293688F5-57D0-468C-88F1-99C1FAAF89B0

\title{
An index to new genera and species of Nematoda in Zootaxa from 2007 to 2012
}

\author{
YU MEI XU ${ }^{1}$, ZENG QI ZHAO ${ }^{1,2 *}$ \& JIAN MING WANG ${ }^{1}$ \\ ${ }^{1}$ Laboratory of Nematology, Department of Plant Pathology, Agronomy College, Shanxi Agricultural University, TaiGu, 030801, \\ China.Email:ymxu0310@yahoo.com.cn; jm.w@sohu.com \\ ${ }^{2}$ Landcare Research, Private Bag 92170, Auckland Mail Centre, Auckland 1142, New Zealand. \\ * Corresponding author:ZhaoZ@landcareresearch.co.nz
}

\begin{abstract}
Of 104 papers on nematodes published in Zootaxa from 2007 to 2012, seventy five, by 136 authors from 27 countries, described eight new genera and 155 new species. A bibliographic analysis of these papers and a list of new genera and species are presented in this paper.
\end{abstract}

Key words: Nematoda, new genera, new species, bibliography, index

\section{Introduction}

Since its establishment in 2001, Zootaxa has become an important journal for publishing new species of animals from all over the world (Zhang 2011). In its first 6 years from 2001 to the end of 2006, 2,388 papers in 52,920 pages were published (Zhao 2007). The first description of a new species of Nematoda in Zootaxa was in 2003 (Pastor de Ward 2003). Zhao (2007) reviewed the new species of Nematoda published in Zootaxa from 2003 to 2006, showing that 17 papers describing 39 new species were published by 38 authors from 13 countries. In its second six years, from 2007 to the end of 2012, a total of 9,016 papers comprising 178,648 pages were published in Zootaxa (http://www.mapress.com/zootaxa/support/Statistics.htm). Obviously Zootaxa significantly expanded in the second six years in terms of paper and page numbers. In addition, 104 papers on nematodes were published in Zootaxa between 2007 and 2012. These included 75 papers on new genera and new species (references are listed with species), 14 on results of surveys (Davies et al. 2010; Gomes et al. 2011; González \& Hamann 2007; Guo et al. 2011; Li et al. 2008; Lunaschi et al. 2012; Paredes-León et al. 2008; Park \& Moraves 2008; Pérez-Álvarez et al. 2008; Shan et al. 2011; Tahseen \& Mustaqim 2012; Venekey et al. 2010; Wouts \& Zhao 2010; Zeng et al. 2012), six with checklists (Justine et al. 2010; Luque et al. 2011; Mata-López et al. 2010; Muniz-Pereira et al. 2009; Zhang et al. 2012; Zhuo et al. 2009), three on molecular taxonomy (Porazinska et al. 2010; Zhao et al. 2012; Zhao \& Buckley 2009), two with taxonomic revisions (Durette-Desset \& Digiani 2010; Peña-Santiago et al. 2012), one editorial paper (Hodda 2011), four monographs (two on new species, one containing a checklist and one on Fergusobia) (Davies et al. 2010; Luque et al. 2011; Paredes-León et al. 2008; Spratt 2011) and four correspondences (Bursey et al. 2007; Gomes et al. 2011; Zhao 2007; Zullini 2012). Therefore, it is interesting to provide a review of the 75 publications of new species of Nematoda in Zootaxa during this period. This paper will summarize the achievements published in Zootaxa from 2007 to 2012, providing a bibliographic analysis of these nematological papers and a list of the new taxa described.

\section{Nematode papers published in Zootaxa, 2007-2012}

Seventy five papers on nematodes were published in Zootaxa between 2007 and 2012: 6 papers (97 pages) in 2007 , 4 (62 pages) in 2008, 20 (326 pages) in 2009, 9 (122 pages) in 2010, 11 (183 pages) in 2011, and 25 (414 pages) in 
2012 (Fig. 1). Eight new genera were reported from six countries: Australia, Germany, Brazil, Spain, Sweden and Russia. One hundred and fifty five new species were reported from 29 countries or oceans: Argentina, Australia, Brazil, Bulgaria, China, Ecuador, France, Germany, Iberia, India, Indonesia, Iran, Kuwait, Mexico, New Caledonia, New Zealand, Papua New Guinea, Norwegian Sea, Russia, Singapore, South Africa, South Korea, Spain, Sweden, Thailand, Vietnam, Atlantic Southeast, East Antarctica and North-Eastern Tropic Pacific.

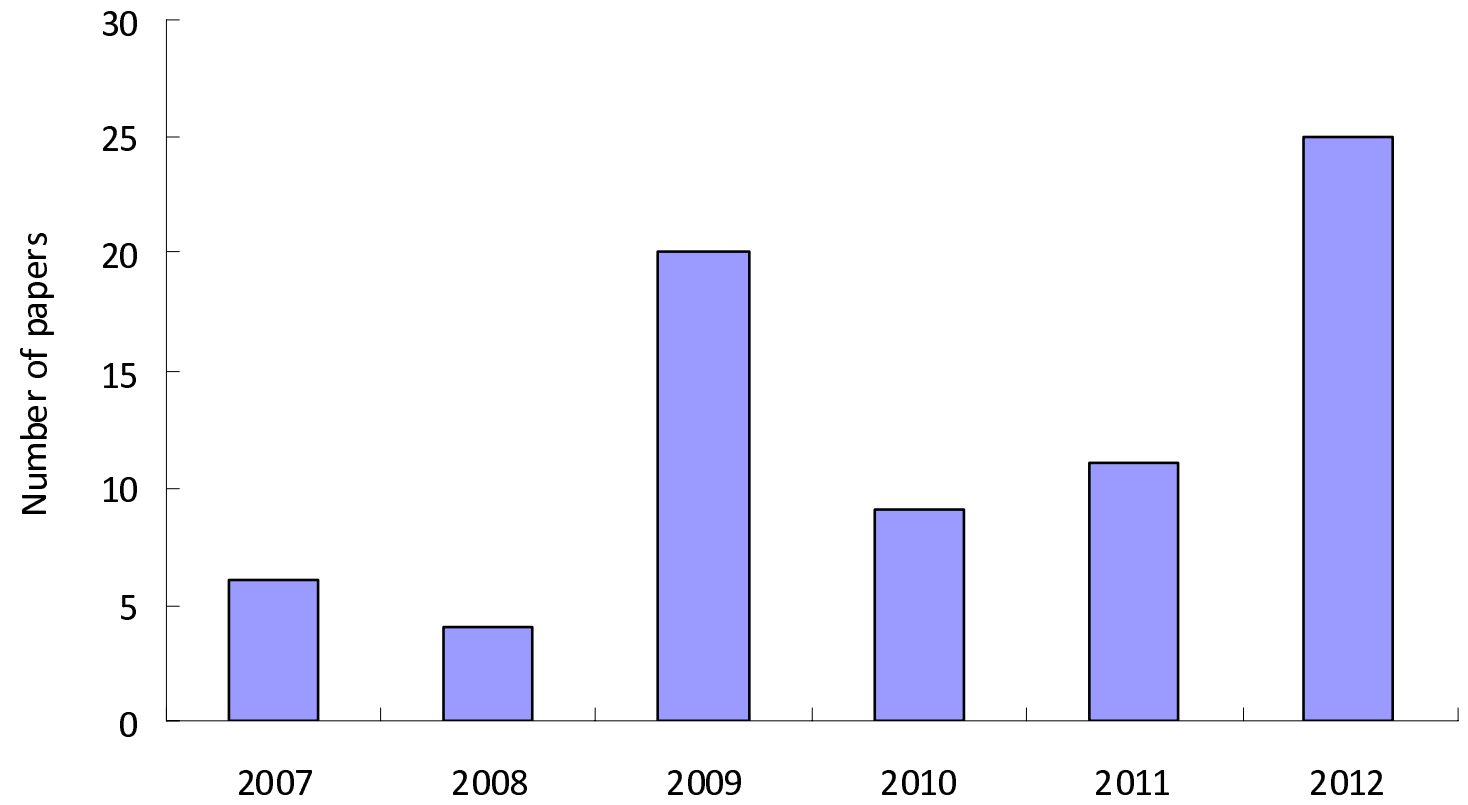

FIGURE 1. Number of papers describing new taxa of Nematoda published in Zootaxa.

Papers ranged in length from 4 to 61 pages, with an average of 16.0 pages. These included 15 papers of less than 10, 43 papers of 10-19 (the majority, Fig. 2), 12 papers of 20-29, and 4 papers of more than 30 pages. The only monograph containing descriptions was of 61 pages.

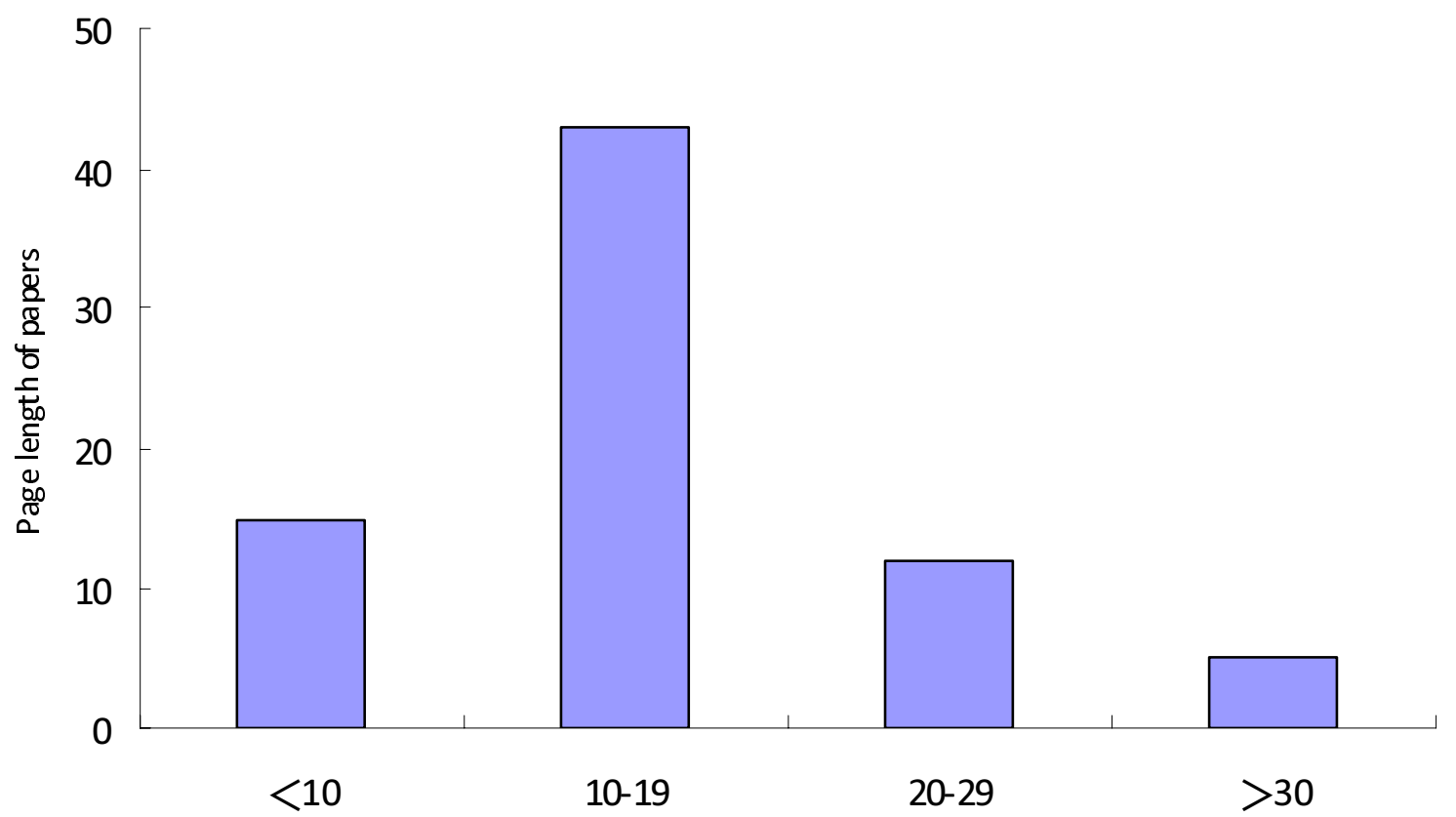

FIGURE 2. Page lengths of papers describing new taxa of Nematoda published in Zootaxa. 
The 75 published papers were contributed by 136 authors from 27 countries: Argentina, Australia, Belgium, Brazil, Bulgaria, China, France, Germany, India, Indonesia, Iran, Italy, Japan, Mexico, New Caledonia, New Zealand, Russia, South Korea, Sweden, Spain, South Africa, The Czech Republic, The Netherlands, Thailand, UK, USA and Vietnam.

The author of the monograph was D. M. Spratt (Australia).

One author, M.C. Silva (Brazil) published six papers. Six authors published four papers: A.M. Esteves (Brazil), E. Pourjam (Iran), J. Abolafia (Spain), V.G. Gagarin (Russia), W. Ahmad (India), and Z.Q. Zhao (New Zealand). Fourteen authors published three papers: H.H. Wang (China) and J.L. Liao (China), K. Davies (Australia), K. Kito (Japan), K. Zhuo (China), L.R. Smales (Australia), L.P. Zhang (China), M.A. Miljutina (Russia), M. Pedram (Iran), M.T. Vinciguerra (Italy), O. Holovachov (Sweden), T.V. Naumova (Russia), V. Fonsêca-Genevois (Brazil) and W.M. Ye (USA).Seventeen authors published two papers: A.P. Botelho (Brazil), C.L. Xu (China), D.M. Miljutin (New Zealand), E.S. Ivanova (Russia), F. Cavalcanti (Brazil), G. Taylor (Australia), H. Xie (China), J. Abolafia (Spain), K. Thomas (USA), M. Baniyamuddin (India), N. Barnes(UK), R. Giblin-Davis (USA), R. Peña-Santiago (Spain), R.Q. Cui (China), S.E. Spiridonov (Russia), T. Chatterjee (India) and V.L. Pham (Vitenam).

Ninety seven authors from 26 countries published one paper, including: Argentina: G. Ramallo; Australia: B. Heinrich, H.J. Weaver and T. Bertozzi; Belgium: A. Vanreusel, N. Smol and W. Decraemer; Brazil: B.C. Guilherme, F.J.V. Castro, L.D.T. Sobral, L. Lins, R.D.C.C. Lima and T.F. Maria; Bulgaria: B.B. Georgiev and Y. Mutafchiev; China: C.B. Zhao, J.X. Zhao, L. Li, L.H. Qiu, M. Luo, M. Zhang, Q.Z. Liu, R.Y. An, S.B. Zhang, S.Q. Zhang, W.J. Liang, X.M. Su, X.N. Hu, X.Y. Li, Y. Li, Y. Pang, Z.D. Wu and Z.Y. Lv; France: B. Odile, I. Beveridge, J. Pierre, L. Jean-Paul, M.C. Durette-Desset and P. Brigitte; Germany: W. Sudhaus; India: A. Chatterjee, A. N. Rizvi, B. Manna, D. Sen, D.K. Gupta, M. Bhaskar, N. Gupta, S. Ahad, T. Naz and U. Tauheed; Indonesia: E. Purwaningsih and K. Dewi; Iran: A. Eskandari, A. Halajian, E. Shokoohi, M.R. Atighi, N. Amirzadi, R. Asghari, R. Ghaemi and R. Heydari; Italy: L. Orselli and M. Clausi; Japan: Y. Ohyama; Mexico: B. MendozaGarfias, G. Parra-Olea, G.P.P. Leon, J.C. Windfield-Perez and J. Falcon-Ordaz; New Caledonia: J.L. Justine; New Zealand: D.A. Wharton, D. Leduc, P.K. Probert and S.D. Nodder; Russia: A.V. Tchesunov, D. Portnova, E.A. Guzeeva and S.V. Kovalyev; South Africa: M.G.J. Hendricks and M.J. Gibbons; Spain: G. Liébanas, R. PeñaSantiago and S. Álvarez-Ortega; Sweden: S. Boström; Thailand: C. Aryuthaka; The Czech Republic: M. Zdeněk, N. Jiří and P. Vladimír; The Netherland: G. Karssen; UK: T. J. Ferrero; USA: C.R. Bursey, D.J. Richardson, K. Kiontke, S.R. Goldberg, S.R. Telford, Jr. and W. D. Hope; South Korea: H.G. Kim and W. Lee.

Of the 75 papers, $13(17.3 \%)$ were published by a single author, $26(34.7 \%)$ by 2 authors, and $36(48 \%)$ by more than 2 authors. In addition, 19 papers (25.3\%) were published by authors from two countries, indicating the collaborative nature of modern nematode taxonomy.

\section{New taxa of Nematoda published in Zootaxa, 2007-2012}

Eight new genera and 155 new species were described in Zootaxa by 136 authors from 27 countries from 2007 to 2012, with 10 new species in 2007, 7 in 2008, 45 in 2009, 23 in 2010, 23 in 2011 and 47 in 2012 (Fig. 3).

\section{Index to new species of Nematoda in Zootaxa, 2007-2012}

Genera and species are listed in alphabetical order and each includes the following information: scientific name, authority, year, page number (country) and family.

\section{New genera: \\ 1. Bathynema Miljutin \& Miljutina, 2009: 24 (Germany)...BENTHIMERMITHIDAE \\ 2. Loveninema Holovachov \& Boström, 2012: 13 (Sweden)...CAMACOLAIMIDAE \\ 3. Mawsonema Smales \& Heinrich, 2010: 28 (Indonesia)...HELIGMONELLIDAE}


4. Montistrongylus Smales \& Heinrich, 2010: 28 (Papua New Guinea)...HELIGMONELLIDAE

5. Paradicelis Ivanova \& Hope, 2009: 15 (Russia)...DRILONEMATIDAE

6. Parasabanema Smales \& Heinrich, 2010: 28 (Papua New Guinea \& Indonesia)...HELIGMONELLIDAE

7. Spirodesma Canti, Silva \& Fonsêca-Genevois, 2009: 10 (Brazil)...DESMODORIDAE

8. Stenodorylaimus Álvarez-Ortega \& Peña-Santiago, 2011, 14 (Spain)...NORDIIDAE

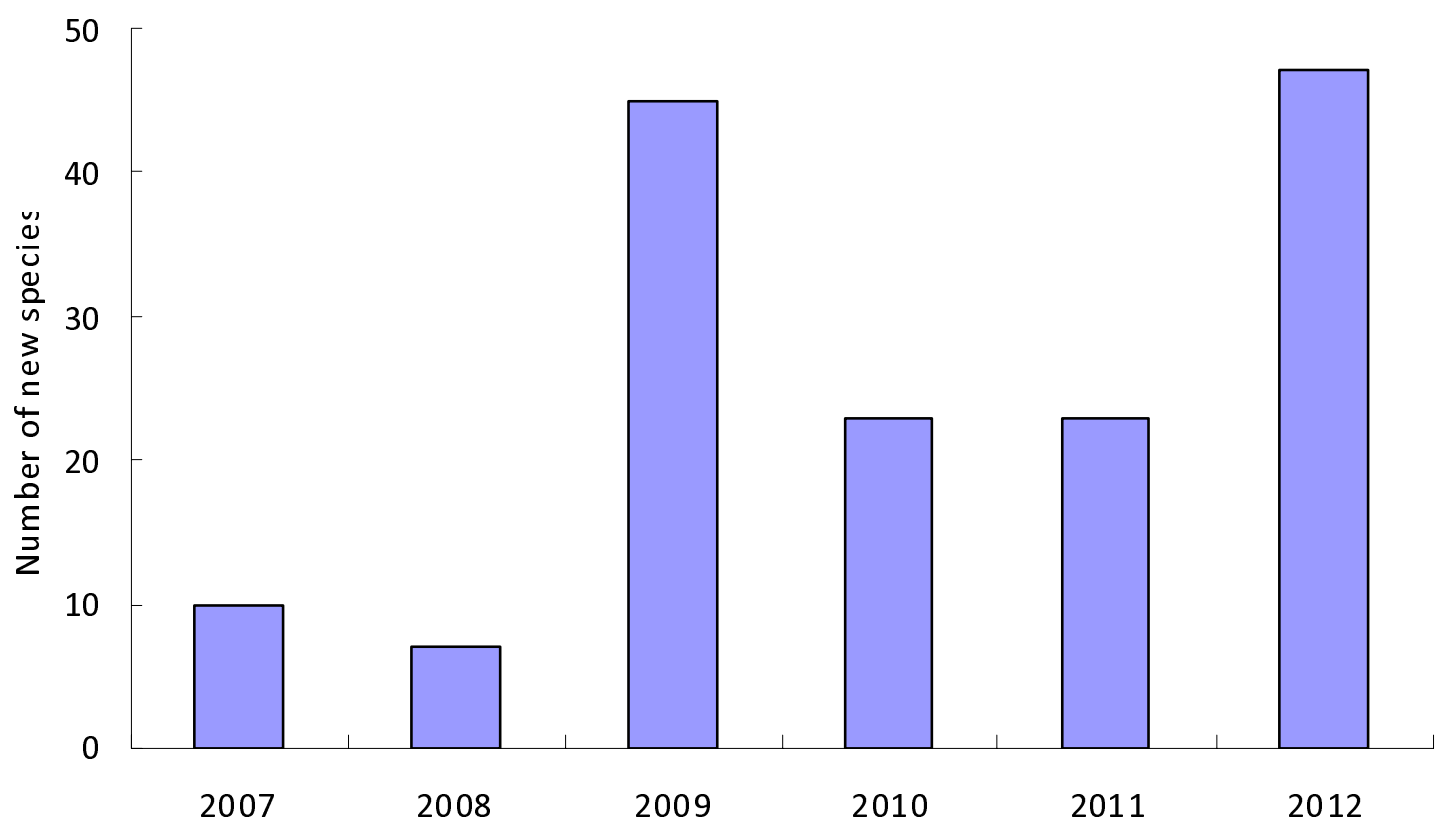

FIGURE 3. Number of new species of Nematoda published in Zootaxa.

\section{New species:}

1. Ablechroiulus dudichi Abolafia \& Peña-Santiago, 2011: 14 (Spain)...RHABDITIDAE

2. Ablechroiulus spelaeus Abolafia \& Peña-Santiago, 2011: 14 (Iberia)...RHABDITIDAE

3. Acrolobus longigubernaculum Amirzadi, Shokoohi, Eskandari \& Abolafia, 2012: 8 (Iran)...CEPHALOBIDAE

4. Anguina paludicola Bertozzi \& Davies, 2009: 14 (Australia)...ANGUINIDAE

5. Antomicron lorenzeni Holovachov, 2012: 15 (Sweden)...LEPTOLAIMIDAE

6. Antomicron quindecimpapillatus Holovachov, 2012: 15 (Sweden)...LEPTOLAIMIDAE

7. Aoruroides cochinchinensis Guzeeva, Pham \& Spiridonov, 2010: 7 (Vietnam)...THELASTOMATIDAE

8. Aphelenchoides fujianensis Zhuo, Cui, Ye, Luo, Wang, Hu \& Liao, 2010: 14 (China)...APHELENCHOIDIDAE

9. Aphelenchoides paradalianensis Cui, Zhuo, Wang \& Liao, 2011: 8 (China)...APHELENCHOIDIDAE

10. Aponema bathyalis Kovalyev \& Miljutina, 2009: 13 (Russia)...MICROLAIMIDAE

11. Aponema martinezi Miljutin \& Miljutina, 2009: 36 (North-Eastern Tropic Pacific)...MICROLAIMIDAE

12. Aponema minutissima Kovalyev \& Miljutina, 2009: 13 (Russia)... MICROLAIMIDAE

13. Aponema ninae Portnova, 2009: 17 (Norwegian Sea)...MICROLAIMIDAE

14. Aponema westindicum Kovalyev \& Miljutina, 2009: 13 (Russia)...MICROLAIMIDAE

15. Aporcelaimellus alpujarrensis Álvarez-Ortega, Abolafia, Liébanas \& Peña-Santiago, 2012: 24 (Iberia)...APORCELAIMIDAE

16. Aporcelaimellus castaneanus Álvarez-Ortega, Abolafia, Liébanas \& Peña-Santiago, 2012: 24 (Iberia)...APORCELAIMIDAE

17. Aporcelaimellus communis Álvarez-Ortega, Abolafia, Liébanas \& Peña-Santiago, 2012: 24 (Iberia)...APORCELAIMIDAE

18. Aporcelaimellus tenuis Álvarez-Ortega, Abolafia, Liébanas \& Peña-Santiago, 2012: 24 (Iberia)...APORCELAIMIDAE

19. Austrostrongylus mawsonae Durette-Desset \& Beveridge, 2012: 41 (Australia)...TRICHOSTRONGYLIDAE

20. Austrostrongylus smalesae Durette-Desset \& Beveridge, 2012: 41 (Australia)...TRICHOSTRONGYLIDAE

21. Axonchium meghalayense Naz \& Ahmad, 2012: 37 (India)...BELONDIRIDAE 
22. Axonchium. noreasum Naz \& Ahmad, 2012: 37 (India)...BELONDIRIDAE

23. Bathynema nodinauti Miljutin \& Miljutina, 2009: 24 (North-Eastern Tropic Pacific)...BENTHIMERMITHIDAE

24. Breinlia (Breinlia) beveridgei Spratt, 2011: 61 (Australia)...FILARIOIDEA

25. Breinlia (Breinlia) bigenera Spratt, 2011: 61 (Australia)...FILARIOIDEA

26. Breinlia (Breinlia) dorcopsis Spratt, 2011: 61 (Australia)...FILARIOIDEA

27. Breinlia (Breinlia) melomyos Spratt, 2011: 61 (Australia)...FILARIOIDEA

28. Breinlia (Breinlia) oweni, Spratt, 2011: 61 (Australia)...FILARIOIDEA

29. Breinlia (Breinlia) presidentei Spratt, 2011: 61 (Australia)...FILARIOIDEA

30. Breinlia (Breinlia) tricondylus Spratt, 2011: 61 (Australia)...FILARIOIDEA

31. Breinlia (Breinlia) zyzomyos Spratt, 2011: 61 (Australia)...FILARIOIDEA

32. Caenorhabditis brenneri Sudhaus \& Kiontke, 2007: 18 (Germany)...RHABDITIDAE

33. Cervonema pseudodeltensis Barnes, Kim. \& Lee, 2012: 28 (South Korea)...COMESOMATIDAE

34. Chromaspirina guanabarensis Maria, Esteves, Smol, Vanreusel \& Decraemer, 2009: 16 (Brazil)...DESMODORIDAE

35. Cosmocephalus pelecani Mutafchiev, Halajian \& Georgiev, 2010: 20 (Australia)...ACUARIIDAE

36. Cosmocephalus podicipis Mutafchiev, Halajian \& Georgiev, 2010: 20 (Iran \& Bulgaria)...ACUARIIDAE

37. Cosmocerca acanthurum Falcon-Ordaz, Windfield-Perez, Mendoza-Garfias \& Leon, 2007: 23 (Mexico)...COSMOCERCIDAE

38. Cosmocercoides karnatakaensis Rizvi, 2009: 11 (India)...COSMOCERCIDAE

39. Cyatholaimus minor Gagarin, 2012: 9 (Vietnam)...CYATHOLAIMIDAE

40. Daptonema hyalocella Aryuthaka \& Kito, 2012: 13 (Thailand)...XYALIDAE

41. Daptonema setihyalocella Aryuthaka \& Kito, 2012: 13 (Thailand)...XYALIDAE

42. Desmodora striatocephala Tchesunov, 2008: 24 (Atlantic Southeast)...CYATHOLAIMIDAE

43. Dicelis eudrilii Ivanova \& Hope, 2009: 15 (Russia)...DRILONEMATOIDEA

44. Discotylenchus iranicus Ghaemi, Pourjam, Atighi, Pedram \& Karssen, 2012: 11 (Iran)...TYLENCHIDAE

45. Distolabrellus magnivulvatus Abolafia \& Peña-Santiago, 2011: 9 (Ibéria)...MESORHABDITIDAE

46. Dolichorhabditis tereticorpus Kito \& Ohyama, 2008: 10 (East Antarctica)...RHABDITIDAE

47. Domorganus suecicus Holovachov, 2012: 6 (Sweden)...OHRIDIIDAE

48. Dorella shamimi Ahmad \& Baniyamuddin, 2010: 8 (Singapore)...TYLENCHOLAIMOIDEA

49. Dorylaimoides alborzicus Pedram, Pourjam \& Vinciguerra, 2011: 11 (Iran)...DORYLAIMOIDINAE

50. Draconema andamanense Kito \& Chatterjee, 2012: 11 (India)...DRACONEMATIDAE

51. Epacanthion agubernaculus Guilherme, Silva \& Esteves, 2009: 10 (Brazil)...THORACOSTOMOPSIDAE

52. Epacrolaimus reyesi Pedram, Pourjam \& Vinciguerra, 2012: 9 (Iran)...APORCELAIMIDAE

53. Ethmolaimus maximus Gagarin \& Naumova, 2012: 10 (Russia)...ETHMOLAIMIDAE

54. Eutobrilus longicaudatoides Gagarin \& Naumova, 2011: 12 (Russia)...TOBRILIDAE

55. Eutobrilus mirandus Gagarin \& Naumova, 2011: 10 (Russia)...TOBRILIDAE

56. Fergusobia camaldulensae Davies, Giblin-Davis, Ye, Taylor \& Thomas, 2012: 22 (Australia)...NEOTYLENCHIDAE

57. Fergusobia eugenioidae Davies, Giblin-Davis, Ye, Taylor \& Thomas, 2012: 36 (Australia)...NEOTYLENCHIDAE

58. Fergusobia fasciculosae Davies, Giblin-Davis, Ye, Taylor \& Thomas, 2012: 36 (Australia) ...NEOTYLENCHIDAE

59. Fergusobia juliae Davies, Giblin-Davis, Ye, Taylor \& Thomas, 2012: 36 (Australia)...NEOTYLENCHIDAE

60. Fergusobia morrisae Davies, Giblin-Davis, Ye, Taylor \& Thomas, 2012: 36 (Australia)... NEOTYLENCHIDAE

61. Fergusobia rileyi Davies, Giblin-Davis, Ye, Taylor \& Thomas, 2012: 22 (Australia)...NEOTYLENCHIDAE

62. Gyrinicola chabadamsoni Brigitte, Odile, Jean-Paul \& Pierre, 2008: 16 (France)...OXYUROIDEA

63. Heterorhabditis beicherriana Li, Liu, Jiří, Vladimír \& Zdeněk, 2012: 16 (China)...HETERORHABDITIDAE

64. Huffmanela ballista Justine, 2007: 19 (New Caledonia)...TRICHOSOMOIDIDAE

65. Huffmanela longa Justine, 2007: 19 (New Caledonia)...TRICHOSOMOIDIDAE

66. Hysterothylacium sinense Li, An \& Zhang, 2007: 10 (China)...ANISAKIDAE

67. Labiobulura (Archeobulura) perditus Smales, 2009: 27 (Australia)...SUBULURIDAE

68. Labiobulura (Labiobulura) quentini Smales, 2009: 27 (Australia)...SUBULURIDAE

69. Laimaphelenchus persicus Asghari Pourjam, Heydari \& Zhao, 2012: 9 (Iran)...APHELENCHOIDIDAE

70. Laimella ferreroi Barnes, Kim. \& Lee, 2012: 28 (South Korea)...COMESOMATIDAE

71. Laimella socotris Barnes, Kim. \& Lee, 2012: 28 (South Korea)...COMESOMATIDAE 
72. Laimella tongyeongensis Barnes, Kim. \& Lee, 2012: 28 (South Korea)...COMESOMATIDAE

73. Laimydorus istvani Sen, Chatterjee \& Manna, 2012: 12 (India)...DORYLAIMOIDEA.

74. Leptepsilonema saldanhae Hendricks \& Gibbons, 2010: 11 (South Africa)...EPSILONEMATIDAE

75. Loveninema tubulosa Holovachov \& Boström, 2012: 13 (Sweden)...CAMACOLAIMIDAE

76. Loveninema unicornis Holovachov \& Boström, 2012: 13 (Sweden)...CAMACOLAIMIDAE

77. Manunema kithara Barnes \& Ferrero, 2009: 16 (Kuwait)...PERESIANIDAE

78. Manunema kuwaitiensis Barnes \& Ferrero, 2009: 16 (Kuwait)...PERESIANIDAE

79. Mawsonema mokwanensis Smales \& Heinrich, 2010: 28 (Indonesia)...HELIGMONELLIDAE

80. Melomystrongylus somoroensis Smales \& Heinrich, 2010: 28 (Papua New Guinea)...HELIGMONELLIDAE

81. Mesodorylaimus bomdillaensis Tauheed \& Ahmad, 2010: 17 (India)...DORYLAIMIDAE

82. Mesodorylaimus khasianus Tauheed \& Ahmad, 2010: 17 (India)...DORYLAIMIDAE

83. Mesodorylaimus noreasus Tauheed \& Ahmad, 2010: 17 (India)...DORYLAIMIDAE

84. Mesodorylaimus spicatus Tauheed \& Ahmad, 2010: 17 (India)...DORYLAIMIDAE

85. Meteterakis wangi Zhang \& Zhang, 2011: 6 (China) ... HETERAKOIDEA

86. Microlaimus abyssalis Miljutin \& Miljutina, 2009: 36 (North-Eastern Tropic Pacific)...MICROLAIMIDAE

87. Microlaimus parviporosus Miljutin \& Miljutina, 2009: 36 (North-Eastern Tropic Pacific)... MICROLAIMIDAE

88. Molgolaimus haakonmosbiensis Portnova, 2009: 17 (Norwegian Sea)...DESMODORIDAE

89. Montistrongylus ingati Smales \& Heinrich, 2010: 28 (Papua New Guinea)...HELIGMONELLIDAE

90. Narsingiella clinotarsi Rizvi, 2009: 11 (India)...OXYURIDAE

91. Neodolichodorus hainanensis Xu, Xie \& Li, 2012: 7 (China)...DOLICHODORIDAE.

92. Neodolichodorus sinensis Zhuo, Wang \& Liao, 2010: 6 (China)...DOLICHODORIDAE

93. Paracanthonchus multisupplementatus Gagarin, 2012: 9 (Vietnam)...CYATHOLAIMIDAE

94. Paracyatholaimus diva Tchesunov, 2008: 24 (Atlantic Southeast)...CYATHOLAIMIDAE

95. Paradicelis bursata Ivanova \& Hope, 2009: 15 (Russia)...DRILONEMATIDAE

96. Paradicelis bursata Ivanova \& Hope, 2009: 15 (Russia)...DRILONEMATOIDEA

97. Paradorylaimus andinus Orselli, Clausi \& Vinciguerra, 2012: 19 (Ecuador)...DORYLAIMIDAE

98. Paradorylaimus flagellicaudatus Orselli, Clausi \& Vinciguerra, 2012: 19 (Ecuador)...DORYLAIMIDAE

99. Paradorylaimus longicaudatus Orselli, Clausi \& Vinciguerra, 2012: 19 (Ecuador)...DORYLAIMIDAE

100. Paradraconema pachylumbus Kito \& Chatterjee, 2012: 11 (India)...DRACONEMATIDAE

101. Paraheligmosomoides amplicaudae Smales \& Heinrich, 2010: 28 (Indonesia)...HELIGMONELLIDAE

102. Paraheligmosomoides ennisae Smales \& Heinrich, 2010: 28 (Papua New Guinea)...HELIGMONELLIDAE

103. Parasabanema szalayi Smales \& Heinrich, 2010: 28 (Papua New Guinea \& Indonesia)...HELIGMONELLIDAE

104. Paramononchus orientalis Gagarin \& Naumova, 2012: 10 (Russia)...MONONCHIDAE

105. Parapharyngodon hemidactylii Gupta, Bhaskar \& Gupta, 2009: 13 (India)...PHARYNGODONIDAE

106. Paraustrostrongylus paramelomysi Smales \& Heinrich, 2010: 28 (Papua New Guinea)...HERPETOSTRONGYLIDAE

107. Perepsilonema benguelae Hendricks \& Gibbons, 2010: 11 (South Africa)...EPSILONEMATIDAE

108. Pharyngonema chinense Ivanova \& Hope, 2009: 15 (Russia)...DRILONEMATOIDEA

109. Pomponema proximamphidum Tchesunov, 2008: 24 (Atlantic Southeast)...CYATHOLAIMIDAE

110. Procyrnea javaensis Zhang, Dewi \& Purwaningsih, 2009: 9 (Indonesia)...HABRONEMATIDAE

111. Prodorylaimus filamentus Gagarin \& Naumova, 2011: 12 (Russia)...DORYLAIMIDAE

112. Pseudochromadora reathae Leduc \& Wharton, 2010: 13 (New Zealand)...DESMODORIDAE

113. Pseudodesmodora lacrima Leduc \& Wharton, 2010: 13 (New Zealand)...DESMODORIDAE

114. Raphidascaris (Sprentascaris) marano Ramallo, 2009:5 (Argentina)...ANISAKIDAE

115. Sabatieria bitumen Botelho, Silva, Esteves \& Fonsêca-Genevois, 2007: 23 (Brazil)...COMESOMATIDAE

116. Sabatieria exilis Botelho, Silva, Sobral \& Fonsêca-Genevois, 2009: 17 (Brazil)...COMESOMATIDAE

117. Sabatieria fidelis Botelho, Silva, Sobral \& Fonsêca-Genevois, 2009: 17 (Brazil)...COMESOMATIDAE

118. Sabatieria paraspiculata Botelho, Silva, Esteves \& Fonsêca-Genevois, 2007: 23 (Brazil)...COMESOMATIDAE

119. Sabatieria spiculata Botelho, Silva, Esteves \& Fonseca-Genevois, 2007: 23 (Brazil)...COMESOMATIDAE

120. Sabatieria subrotundicauda Botelho, Silva, Esteves \& Fonseca-Genevois, 2007: 23

(Brazil)...COMESOMATIDAE

121. Scutylenchus dongtingensis Xu, Xie, Zhao, Zhang \& Su, 2012 :11 (China)...MERLINIINAE

122. Setosabatieria conicauda Probert \& Nodder, 2012: 16 (New Zealand)...COMESOMATIDAE

123. Siconema diducuncinum Ivanova, Pham \& Spiridonov, 2012:13 (Russia)...UNGELLIDAE 
124. Siconema ovicallosum Ivanova, Pham \& Spiridonov, 2012: 13 (Russia)...UNGELLIDAE

125. Spauligodon xenosauri Bursey, Goldberg \& Telford, 2007: 4 (Mexico)...PHARYNGODONIDAE

126. Spirinia lara Silva, Castro, Cavalcanti \& Fonsêca-Genevois, 2009: 15 (Brazil)...DESMODORIDAE

127. Spirinia sophia Silva, Castro, Cavalcanti \& Fonsêca-Genevois, 2009: 15 (Brazil)...DESMODORIDAE

128. Spirodesma magdae Canti, Silva \& Fonsêca-Genevois, 2009: 10 (Brazil)...DESMODORIDAE

129. Sprattia spearei Spratt, 2011: 61 (Australia)...FILARIOIDEA

130. Steinernema pui Qiu, Zhao, Wu, Lv \& Pang, 2011: 13 (China)...STEINERNEMATIDAE

131. Syphacia (Syphacia) brevicaudata Weaver \& Smales, 2008: 12 (Australia)...OXYURIDAE

132. Syphacia (Syphacia) pseudomyos Weaver \& Smales, 2008: 12 (Australia)...OXYURIDAE

133. Syringolaimus annae Lima, Lins, Silva \& Esteves, 2009: 18 (Brazil)...IRONIDAE

134. Syringolaimus magdae Lima, Lins, Silva \& Esteves, 2009: 18 (Brazil)...IRONIDAE

135. Syringolaimus smolae Lima, Lins, Silva \& Esteves, 2009: 18 (Brazil)...IRONIDAE

136. Syringolaimus taniae Lima, Lins, Silva \& Esteves, 2009: 18 (Brazil)...IRONIDAE

137. Tantunema enigmatum Ahmad \& Baniyamuddin, 2010: 8 (Singapore)...TYLENCHOLAIMOIDEA

138. Torquatoides noerdjitoi Zhang, Dewi \& Purwaningsih, 2009: 9 (Indonesia)...HABRONEMATIDAE

139. Tripyla bioblitz Zhao, 2009: 16 (New Zealand)...TRIPYLIDAE

140. Tripylina kaikoura Zhao, 2009: 24 (New Zealand)...TRISCHISTOMATIDAE

141. Tripylina manurewa Zhao, 2009: 24 (New Zealand)...TRISCHISTOMATIDAE

142. Tripylina tamaki Zhao, 2009: 24 (New Zealand)...TRISCHISTOMATIDAE

143. Tripylina tearoha Zhao, 2009: 24 (New Zealand)...TRISCHISTOMATIDAE

144. Tripylina yeatesi Zhao, 2009: 24 (New Zealand)...TRISCHISTOMATIDAE

145. Trischistoma otaika Zhao, 2011: 25 (New Zealand)...TRISCHISTOMATIDAE

146. Trischistoma triregius Zhao, 2011: 25 (New Zealand)...TRISCHISTOMATIDAE

147. Trischistoma tukorehe Zhao,2011: 25 (New Zealand)...TRISCHISTOMATIDAE

148. Trischistoma waiotama Zhao, 2011: 25 (New Zealand) ... TRISCHISTOMATIDAE

149. Trophomera elegantis Miljutin \& Miljutina, 2009: 24 (North-Eastern Tropic Pacific)...BENTHIMERMITHIDAE

150. Trophomera minutissima Miljutin \& Miljutina, 2009: 24 (North-Eastern Tropic Pacific)...BENTHIMERMITHIDAE

151. Trophomera pacifica Miljutin \& Miljutina, 2009: 24 (North-Eastern Tropic Pacific)...BENTHIMERMITHIDAE

152. Trophomera senckenbergi Miljutin \& Miljutina, 2009: 24 (North-Eastern Tropic Pacific)...BENTHIMERMITHIDAE

153. Tylencholaimellus sinensis Zhang, Ahad, Baniyamuddin, Liang \& Ahmad, 2012: 17 (China)...LEPTONCHIDAE

154. Tylencholaimus singaporensis Ahmad \& Baniyamuddin, 2010: 8 (Singapore)...TYLENCHOLAIMOIDEA

155. Vasostoma aurata Leduc, Probert \& Nodder, 2012: 16 (New Zealand)...COMESOMATIDAE

\section{References}

Abolafia, J. \& Peña-Santiago, R. (2011) Ablechroiulus spelaeus sp. n. and A. dudichi Andrássy, 1970 from Andalucía Oriental, Spain, with a discussion of the taxonomy of the genus Ablechroiulus Andrássy, 1966 (Nematoda, Rhabditida, Rhabditidae). Zootaxa, 2922, 1-14 (17 June).

Abolafia, J. \& Peña-Santiago, R. (2011) Description of Distolabrellus magnivulvatus sp. n. (Nematoda, Rhabditida, Mesorhabditidae) from Iberian peninsula, the second species of a rare genus. Zootaxa, 2804, 56-64 (30 March).

Ahmad, W. \& Baniyamuddin, M. (2010) Three new and a known species of Tylencholaimoidea (Nematoda: Dorylaimida) from Singapore. Zootaxa, 2505, 51-64 (14 June).

Álvarez-Ortega S. \& Peña-Santiago R. (2012) Re-description of two atypical species of Pungentus Thorne \& Swanger, 1936, with proposal of Stenodorylaimus gen. n. (Nematoda, Dorylaimida, Nordiidae). Zootaxa, 2799, 49-62 (24 March).

Álvarez-Ortega, S., Abolafia, J., Liébanas, G. \& Peña-Santiago, R. (2012) Studies on the genus Aporcelaimellus Heyns, 1965 (Nematoda, Dorylaimida, Aporcelaimidae). Four new species with complex uterus from Southeastern Iberian Peninsula. Zootaxa, 3551, 1-24 (16 November).

Amirzadi, N., Shokoohi, E., Eskandari, A. \& Abolafia, J. (2012) Description of Acrolobus longigubernaculum sp. n. (Nematoda, Rhabditida, Cephalobidae) from Iran, the second species of the genus. Zootaxa, 3407, 61-68 (2 August).

Aryuthaka, C. \& Kito, K. (2012) Two new species of the genus Daptonema Cobb, 1920 (Nematoda: Xyalidae) found in the monospecific Halophila ovalis patches within an intertidal mixed-species seagrass bed on the coast of the Andaman Sea, Thailand. Zootaxa, 3350, 34-45 (19 June).

Asghari, R., Pourjam, E., Heydari R. \& Zhao, Z.Q. (2012) Laimaphelenchus persicus n. sp. (Nematoda: Aphelenchoididae) 
from Iran. Zootaxa, 3325, 59-67 (25 May).

Barnes, N. \& Ferrero, T.J. (2009) Two new species of Manunema (Plectida: Peresianidae) from the Arabian Gulf, with notes on the phylogeny of the genus. Zootaxa, 2053, 43-58 (25 March).

Barnes, N., Kim, H.G. \& Lee, W. (2012) New species of free-living marine Sabatieriinae (Nematoda: Monhysterida: Comesomatidae) from around South Korea. Zootaxa, 3368, 263-290 (4 July).

Bertozzi, T. \& Davies, K.A. (2009) Anguina paludicola sp. n. (Tylenchida: Anguinidae): The nematode associated with Rathayibacter toxicus infection in Polypogon monspeliensis and Lachnagrostis filiformis in Australia. Zootaxa, 2060, 3346 (1 April).

Botelho, A.P., Silva, M.C., Esteves, A.M., Fonseca-Genevois, V. (2007) Four new species of Sabatieria Rouville, 1903 (Nematoda, Comesomatidae) from the Continental Slope of Atlantic Southeast. Zootaxa, 1402, 39-57 (1 February).

Botelho, A.P., Silva, M.C., Sobral, L.D.T. \& Fonsêca-Genevois, V. (2009) Two new species of Sabatieria Rouville (Nematoda: Comesomatidae) with conical-cylindrical tails, from Campos Basin, Rio de Janeiro, Brazil. Zootaxa, 2096 , 82-98 (11 May).

Brigitte, P., Odile, B., Jean-Paul, L. \& Pierre, J. (2008) Gyrinicola chabadamsoni n. sp. and G. tba (Dinnik 1933) (Nematoda, Oxyuroidea) from tadpoles of the hybridogenetic complex Rana lessonae-esculenta (Amphibia, Ranoidea). Zootaxa, 1764, 25-40 (7 May).

Bursey, C.R., Goldberg, S.R. \& Telford, Jr. S.R. (2007) New species of Spauligodon (Nematoda: Pharyngodonidae) in Xenosaurus platyceps (Squamata: Xenosauridae) from Mexico. Zootaxa, 1501, 65-68 (7 June).

Cavalcanti, M.D.F., Silva, M.C. \& Fonsêca-Genevois, V. (2009) Spirodesma magdae nov. gen. nov. sp. (Nematoda: Desmodoridae) from the Brazilian deep sea (Campos Basin, Rio de Janeiro, Brazil). Zootaxa, 2096, 109-118 (11 May).

Cui, R.Q., Zhuo, K., Wang, H.H. \& Liao, J.L. (2011) Aphelenchoides paradalianensis n. sp. (Nematoda: Aphelenchoididae) isolated at Guangzhou, China, in packaging wood from South Korea. Zootaxa, 2864, 57-64 (3 May).

Davies K.A., Giblin-Davis, R.M., Ye, W.M., Taylor, G.S. \& Thomas W.K. (2010) Nematodes from galls on Myrtaceae. I. Fergusobia/Fergusonina galls on Corymbia spp., with re-description of $F$. magna and notes on its phylogenetic relationships. Zootaxa, 2634, 25-40 (4 October).

Davies, K., Giblin-Davis, R., Ye, W.M., Taylor, G. \& Thomas, K. (2012) Nematodes from galls on Myrtaceae. II. Fergusobia/ Fergusonina from small axillary bud ('stem') and leaf ('pea') galls in Australia, with descriptions of two new species. Zootaxa, 3415, 1-22 (9 August).

Davies, K., Giblin-Davis, R., Ye, W.M., Taylor, G. \& Thomas, K. (2012) Nematodes from galls on Myrtaceae. III. Fergusobia from flower bud and stigma galls on Eucalyptus, with descriptions of four new species. Zootaxa, 3532, 1-36 (31 October).

Davies, K., Ye, W.M., Giblin-Davis, R., Taylor, G., Scheffer, S. \& Thomas, K. (2010) The nematode genus Fergusobia (Nematoda: Neotylenchidae): molecular phylogeny, descriptions of clades and associated galls, host plants and Fergusonina fly larvae. Zootaxa, 2633, 1-66 (1 October).

Durette-Desset, M.C. \& Beveridge, I. (2012) Redescriptions and descriptions of new species of Austrostrongylus Chandler, 1924 (Nematoda: Trichostrongylina), from Australian marsupials with a comparative study of features of the synlophe. Zootaxa, 3512, 1-41 (10 October).

Durette-Desset, M. \& Digiani, M.C. (2010) Taxonomic revision of the type specimens of Ethiopian Nippostrongylinae (Nematoda) deposited at the Natural History Museum of London. Zootaxa, 2494, 1-28 (4 June).

Falcon-Ordaz, J., Windfield-Perez, J.C., Mendoza-Garfias, B., Parra-Olea, G. \& De Leon, G.P.P. (2007) Cosmocerca acanthurum n. sp. (Nematoda: Cosmocercidae) in Pseudoeurycea leprosa and Chiropterotriton orculus from the Transmexican Volcanic Belt, Central Mexico, with a checklist of the helminth parasites of plethodontid salamanders. Zootaxa, 1434, 27-49 (29 March).

Gagarin, V.G. \& Naumova, T.V. (2011) Prodorylaimus filamentus sp. n. and Eutobrilus longicaudatoides sp. n. (Nematoda) from Lake Baikal, Russia. Zootaxa, 3103, 57-68 (18 November).

Gagarin, V.G. \& Naumova,T.V. (2012) Paramononchus orientalis sp. n. and Ethmolaimus maximus sp. n. (Nematoda) from Lake Baikal, Russia. Zootaxa, 3513: 51-60 (10 October).

Gagarin, V.G. (2012) Paracanthonchus multisupplementatus sp. n. and Cyatholaimus minor sp. n. (Nematoda) from the coast of Vietnam. Zootaxa, 3392, 60-68 (18 July).

Gagarin. V.G. \& Naumova, T.V. (2011) Two nematode species of the family Tobrilidae (Enoplida) De Coninck, 1965 from Lake Baikal. Russia, Zootaxa, 2815, 59-68 (12 April).

Ghaemi, R., Pourjam, E., Atighi, M.R., Pedram, M. \& Karssen, G. (2012) First record of the genus Discotylenchus Siddiqi, 1980 (Nematoda: Tylenchidae) from Iran, with description of one new and data on two known species. Zootaxa, 3493, 7282 (21 September).

Gomes, S.N., Pesenti, T.C., Aguiar, C.L.G. \& Müller, G. (2011) Nematodes of the nine-banded armadillo, Dasypus novemcinctus (Xenarthra: Dasypodidae) in Rio Grande do Sul, Brazil. Zootaxa, 3099, 65-68 (16 November).

González, C.E. \& Hamann, M.I. (2007) Nematode parasites of two species of Chaunus (Anura: Bufonidae) from Corrientes, Argentina. Zootaxa, 1393, 27-34 (18 Jane).

Guilherme, B.C., Silva, M.C. \& Esteves, A.M. (2009) Description of a new species of Epacanthion (Thoracostomopsidae, Nematoda) from Brazil and a modified key for species identification. Zootaxa, 2096, 99-108 (11 May).

Guo, K., Shi, H.L., Liu, K. \& Zheng, J.W. (2011) Past and present distribution and hosts of Longidorus (Nematoda: Dorylaimida) in mainland China. Zootaxa, 3086, 27-38 (02 November). 
Gupta, N., Bhaskar, M. \& Gupta, D.K. (2009) Gastro-intestinal invasion in Hemidactylus flaviviridis with a new species of Parapharyngodon (Oxyuroidea: Pharyngodonidae). Zootaxa, 2165, 39-51 (22 July).

Guzeeva, E.A., Pham, V.L. \& Spiridonov, S.E. (2010) Aoruroides cochinchinensis sp. n. (Oxyurida: Thelastomatidae) from Vietnamese Panesthiinae wood-burrowing cockroaches. Zootaxa, 2477, 62-68 (19 May).

Hendricks, M.G.J. \& Gibbons, M.J. (2010) Two new nematode species from Saldanha Bay, South Africa: Perepsilonema benguelae sp. nov. and Leptepsilonema saldanhae sp. nov. (Desmodorida, Epsilonematidae). Zootaxa, 2504, 20-30 (14 June).

Hodda, M. (2011) Phylum Nematoda Cobb 1932. In: Zhang, Z.-Q. (Ed.) Animal biodiversity: An outline of higher-level classification and survey of taxonomic richness. Zootaxa, 3148, 63-95 (23 December).

Holovachov, O. (2012) Swedish Plectida (Nematoda). Part 1. Domorganus suecicus sp. n. from Skagerrak. Zootaxa, 3263, 6368 (9 April).

Holovachov, O. (2012) Swedish Plectida (Nematoda). Part 2. The genus Antomicron Cobb, 1920. Zootaxa, 3380, 39-54 (5 July).

Holovachov, O. \& Boström, S. (2012) Swedish Plectida (Nematoda). Part 3. The genus Loveninema gen. n. Zootaxa, 3505, 2638 (3 October).

Ivanova, E.S., Pham, V.L. \& Spiridonov, S.E. (2012) Two new species of Siconema (Drilonematoidea: Ungellidae) parasitic in earthworms in Vietnam, and systematic relationships as inferred from ribosomal sequence data. Zootaxa, 3344, 47-59 (13 June).

Ivanova, E.S. \& Hope, W.D. (2009) A new genus and three new species of Drilonematidae (Rhabditida, Drilonematoidea) from earthworms. Zootaxa, 2293, 53-67 (19 November).

Justine, J.L. (2007) Huffmanela spp. (Nematoda, Trichosomoididae) parasites in coral reef fishes off New Caledonia, with descriptions of $H$. balista n. sp. and H. longa n. sp. Zootaxa, 1628, 23-41 (2 November).

Justine, J., Beveridge, I., Boxshall, G.A., Bray, R.A., Moravec, F. \& Whittington, I.D. (2010) An annotated list of fish parasites (Copepoda, Monogenea, Digenea, Cestoda and Nematoda) collected from Emperors and Emperor Bream (Lethrinidae) in New Caledonia further highlights parasite biodiversity estimates on coral reef fish. Zootaxa, 2691, 1-40 (30 November).

Kito, K. \& Ohyama, Y. (2008) Rhabditid nematodes found from a rocky coast contaminated with treated wastewater of Casey Station in East Antarctica, with a description of a new species of Dolichorhabditis Andrassy, 1983 (Nematoda: Rhabditidae). Zootaxa, 1850, 43-52 (15 August).

Kito, K. \& Chatterjee, T. (2012) New species of the genera Draconema Cobb, 1913 and Paradraconema Allen \& Noffsinger, 1978 (Nematoda: Draconematidae) from the Andaman Islands, Indian Ocean, with keys to the species. Zootaxa, 3575, 7888 (7 December).

Kovalyev, S.V. \& Miljutina, M.A. (2009) A review of the genus Aponema Jensen, 1978 (Nematoda: Microlaimidae) with description of three new species. Zootaxa, 2077, 56-68 (20 April).

Leduc, D., Probert, P.K. \& Nodder, S.D. (2012) Two new free-living nematode species (Comesomatidae) from the continental slope of New Zealand, with keys and notes on distribution. Zootaxa, 3348, 40-55 (15 June).

Li, L., An, R.Y. \& Zhang, L.P. (2007) A new species of Hysterothylacium (Nematoda: Anisakidae) from marine fishes from Yellow Sea, China, with a key to the species of the genus Hysterothylacium. Zootaxa, 1614, 43-52 (15 October).

Li, L. Xu, Z. \& Zhang, L.P. (2008) Redescription of three species of Hysterothylacium (Nematoda: Anisakidae) from marine fishes from the Yellow Sea, China, with the synonymy of Hysterothylacium muraenesoxin (Luo, 1999). Zootaxa, 1878, 55-67 (17 September).

Li, X.Y., Liu, Q.Z., Jiří, N., Vladimír, P. \& Zdeněk M. (2012) Heterorhabditis beicherriana n. sp. (Nematoda: Heterorhabditidae), a new entomopathogenic nematode from the Shunyi district of Beijing, China. Zootaxa, 3569, 25-40 (30 November).

Lima, R.D.C.C., Lins, L., Silva, M.C. \& Esteves, A.M. (2009) Four new species of Syringolaimus De Man, 1888 (Nematoda: Ironidae) from the Southeast Atlantic (Brazil), with redefinition of valid species and the proposal of a new key. Zootaxa, 2096, 119-136 (11 May).

Leduc, D. \& Wharton, D.A. (2010) New free-living marine nematode species (Nematoda: Desmodoridae) from the coast of New Zealand. Zootaxa, 2611, 45-57 (14 September).

Lunaschi, L.I., Álvarez, V.H.M. \& Damborenea, C. (2012) Type material housed in the Helminthological Collection of the Museo de La Plata, Buenos Aires, Argentina. Zootaxa, 3199, 1-59 (20 February).

Luque, J.L., Aguiar, J.C., Vieira, F.M., Gibson, D.I. \& Santos, C.P. (2011) Checklist of Nematoda associated with the fishes of Brazil. Zootaxa, 3082, 1-88 (28 October).

Maria, T.F., Esteves, A.M., Smol, N., Vanreusel, A. \& Decraemer, W. (2009) Chromaspirina guanabarensis sp. n. (Nematoda: Desmodoridae) and a new illustrated dichotomous key to Chromaspirina species. Zootaxa, 2092, 21-36 (6 May).

Mata-López, R., García-Prieto, L. \& León-Règagnon, V. (2010) Helminths of the American bullfrog, Lithobates catesbeianus (Shaw, 1802), from Pawnee Lake, Lancaster, Nebraska, USA with a checklist of its helminth parasites. Zootaxa, 2544, 153 (21 July).

Miljutin, D.M. \& Miljutina, M.A. (2009) Deep-sea nematodes of the family Microlaimidae from the Clarion-Clipperton Fracture Zone (North-Eastern Tropic Pacific), with the descriptions of three new species. Zootaxa, 2096, $137-172$ (11 May).

Miljutin, D.M. \& Miljutina, M.A. (2009) Description of Bathynema nodinauti gen. n., sp. n. and four new Trophomera species 
(Nematoda: Benthimermithidae) from the Clarion-Clipperton Fracture Zone (Eastern Tropic Pacific), supplemented with the keys to genera and species. Zootaxa, 2096, 173-196 (11 May).

Mutafchiev, Y., Halajian, A. \& Georgiev, B.B. (2010) Two new nematode species of the genus Cosmocephalus Molin, 1858 (Spirurida: Acuariidae), with an amended generic diagnosis and an identification key to Cosmocephalus spp. Zootaxa, 2349, 1-20 (2 February).

Muniz-Pereira, L.C., Vieira, F.M. \& Luque, J.L. (2009) Checklist of helminth parasites of threatened vertebrate species from Brazil. Zootaxa 2123, 1-45 (2 June).

Naz, T. \& Ahmad, W. (2012) Description of two new and five known species of the genus Axonchium Cobb, 1920 (Nematoda : Dorylamida) from India with diagnostic compendia and keys to species of the genera Axonchium and Syncheilaxonchium Coomans \& Nair, 1975. Zootaxa, 3264, 1-37 (10 April).

Orselli, L., Clausi, M. \& Vinciguerra, M. T. (2012) The genus Paradorylaimus Andrássy, 1969 (Nematoda: Dorylaimida) with description of three new species from Ecuador. Zootaxa, 3302, 25-43 (7 May).

Paredes-León, R., García-Prieto, L., Guzmán-Cornejo, C., León-Règagnon, V. \& Pérez, T.M. (2008) Metazoan parasites of Mexican amphibians and reptiles. Zootaxa, 1904, 1-166 (15 October).

Pérez-Álvarez, Y., García-Prieto, L., Osorio-Sarabia, D., Lamothe-Argumedo, R. \& León-Règagnon, V. (2008) Present distribution of the genus Gnathostoma (Nematoda: Gnathostomatidae) in Mexico. Zootaxa, 1930, 39-55 (12 November).

Park, J. \& Moravec, F. Redescription of Cucullanus robustus (Nematoda: Cucullanidae) from the conger eel Conger myriaster off Korea. Zootaxa, 1729, 1-7 (20 March).

Pastor de Ward, C.T. (2003) Two new species of Sabatieria (Nematoda, Comesomatidae) from Golfo Nuevo, Chubut (Argentina). Zootaxa, 172, 1-12 (31 March).

Pedram, M., Pourjam, E. \& Vinciguerra, M.T. (2012) Description of a new species of the rare genus Epacrolaimus Andrássy, 2000 (Dorylaimida, Aporcelaimidae) and new data on male of Paraxonchium laetificans (Andrássy, 1956) Altherr \& Loof, 1969 (Dorylaimida, Paraxonchiidae) from Iran. Zootaxa, 3327, 53-61 (29 May).

Pedram, M., Pourjam, E. \& Vinciguerra, M.T. (2011) Description of Dorylaimoides alborzicus sp. n. (Dorylaimida: Nematoda) from Iran, with updated compendium and key to the species of Dorylaimoides. Zootaxa, 3022, 58-68 (12 September).

Peña-Santiago, R., Abolafia, J. \& Pedram, M. (2012) New data on Labronemella labiata Andrássy, 1985 (Dorylaimida: Qudsianematidae) from Iran, with SEM study and a compendium of the genus. Zootaxa, 3271, 43-54 (18 April).

Porazinska, D., Giblin-Davis, R., Sung, W. \& Thomas, W. (2010) Linking operational clustered taxonomic units (OCTUs) from parallel ultra sequencing (PUS) to nematode species. Zootaxa, 2427, 55-63 (15 April).

Portnova, D. (2009) Free-living nematodes from the deep-sea Håkon Mosby Mud Volcano, including the description of two new and three known species. Zootaxa, 2096, 197-213 (11 May).

Qiu, L.H., Zhao, J.X., Wu, Z.D., Lv, Z.Y. \& Pang, Y. (2011) Steinernema pui sp. n. (Rhabditida, Steinernematidae), a new entomopathogenic nematode from Yunnan, China. Zootaxa, 2767, 1-13 (17 February).

Ramallo, G. (2009) A new species of Raphidascaris (Sprentascaris) (Nematoda: Anisakidae) in Hypostomus cordovae (Pisces: Loricariidae) from Argentina. Zootaxa, 2045, 60-64 (18 March).

Rizvi, A.N. (2010) Two new species of amphibian nematodes from Bhadra Wildlife Sanctuary, Western Ghats, India. Zootaxa, 2013, 58-68 (16 February).

Sen, D., Chatterjee, A. \& Manna, B. (2012) One new and two known species of Dorylaimoidea (Nematoda) from West Bengal, India. Zootaxa, 3258, 46-57(5 April).

Shah, M.M., Mohilal, N., Pramodini, M., Bina, L. \& Hemananda, T. (2011) Nematode parasites of invertebrates from Manipur, North East India (Diagnosis, keys and illustration). Zootaxa, 3107, 01-37 (23 November).

Silva, M.C., Castro, F.J.V., Cavalcanti, M.D.F. \& Fonsêca-Genevois,V. (2009) Spirinia lara sp. n. and Spirinia sophia sp. n. (Nematoda, Desmodoridae) from the Brazilian continental margin (Campos Basin, Rio de Janeiro). Zootaxa, 2081, 31-45 (24 April).

Smales, L.R. \& Heinrich, B. (2009) Gastrointestinal nematodes of Paramelomys rubex (Rodentia: Muridae) from Papua Indonesia and Papua New Guinea with the descriptions of three new genera and four new species of Helligmonellidae and Herpetostrongylidae (Nematoda: Trichostrongylida). Zootaxa, 2672, 1-28 (10 November).

Smales, L.R. (2009) A review of the nematode genus Labiobulura (Ascaridida: Subuluridae) parasitic in bandicoots (Peramelidae) and bilbies (Thylocomyidae) from Australia and rodents (Murinae: Hydromyini) from Papua New Guinea with the description of two new species. Zootaxa, 2209, 1-27 (26 August).

Spratt, D. M. (2011) New records of filarioid nematodes (Nematoda: Filarioidea) parasitic in Australasian monotremes, marsupials and murids, with descriptions of nine new species. Zootaxa, 2860, 1-61 (29 April).

Sudhaus, W. \& Kiontke, K. (2007) Comparison of the cryptic nematode species Caenorhabditis brenneri sp. n. and $C$. remanei (Nematoda: Rhabditidae) with the stem species pattern of the Caenorhabditis elegans group. Zootaxa, 1456, 45-62 (25 April).

Tahseen, Q. \& Mustaqim, M. (2012) Descriptions of six known species of Plectus Bastian, 1865 (Nematoda, Plectida, Plectidae) from India with a discussion on the taxonomy of the genus. Zootaxa, 3205, 1-25 (24 Feburary).

Tauheed, U. \& Ahmad, W. (2010) Four new species of the genus Mesodorylaimus Andrássy, 1959 (Nematoda: Dorylaimida) from India. Zootaxa, 2642, 19-35 (12 October).

Tchesunov, A.V. (2008) Three new species of free-living nematodes from the South-East Atlantic Abyss (DIVA I Expedition). Zootaxa, 1866, 151-174 (3 September). 
Venekey, V., Fonseca-Genevois, V. \& Santos, P.J.P. (2010) Biodiversity of free-living marine nematodes on the coast of Brazil: a review. Zootaxa, 2568, 39-66 (16 August).

Weaver, H.J. \& Smales, L.R. (2008) New species of Syphacia (Syphacia) Seurat (Nematoda: Oxyuridae) from Pseudomys species (Rodentia: Muridae) from central Australia. Zootaxa, 1775, 39-50 (23 May).

Wouts, W.M. \& Zhao, Z.Q. (2010) Type material in the National Nematode Collection of New Zealand. Zootaxa, 2611, 58-68 (14 September).

Xu, C.L., Xie, H. \& Li, Y. (2012) Description of Neodolichodorus hainanensis n. sp. (Nematoda: Dolichodoridae) from rhizosphere soil of golf turf in China. Zootaxa, 3236, 62-68(16 March).

Xu, C.L., Xie, H., Zhao, C. B., Zhang, S.B. \& Su, X.M. (2012) Review of the genus Scutylenchus Jairajpuri, 1971 (Nematoda: Tylenchida), with description of Scutylenchus dongtingensis n. sp. from rhizosphere soil of grass in China. Zootaxa, 3437, 32-42 (24 August).

Zeng, Y.S., Ye, W.M., Tredway, L., Martin, S. \& Martin, M. (2012) Taxonomy and morphology of plant-parasitic nematodes associated with turfgrasses in North and South Carolina, USA. Zootaxa, 3452, 1-46 (4 September).

Zhang, L.P., Dewi, K. \& Purwaningsih, E. (2009) Two new species of habronematid nematodes (Nematoda: Spirurida: Habronematidae) in birds of prey from West Java, Indonesia with a key to species of Torquatoides. Zootaxa, 2290, 50-58 (16 November).

Zhang, M., Ahad, S., Baniyamuddin, M., Liang, W.J. \& Ahmad, W. (2012) A new and three known species of the genus Tylencholaimellus Cobb in M.V. Cobb, 1915 (Nematoda: Dorylaimida) from Changbai Mountain, China. Zootaxa, 3499, 46-62 (27 September).

Zhang, S.Q, Bu, Y.Z., Huang, G.P., Wen, Q. \& Zhang, L.P. (2012) A checklist of parasitic nematodes (Nematoda) from birds (Aves) in China. Zootaxa, 3446, 1-31 (30 August).

Zhang, S.Q. \& Zhang, L.P. (2011) A new species of Meteterakis Karve, 1930 (Nematoda: Heterakoidea) from Indotestudo elongata (Blyth) in China with a key to the species of Meteterakis. Zootaxa, 2869, 63-68 (6 May).

Zhang, Z.Q. (2011) Accelerating biodiversity descriptions and transforming taxonomic publishing: the first decade of Zootaxa. Zootaxa, 2896, 1-7 (28 May).

Zhao, Z.Q. (2009) A review of the genus Tripylina Brzeski, 1963 (Nematoda: Triplonchida), with descriptions of five new species from New Zealand. Zootaxa, 2238, 1-24 (23 September). Zhao, Z.Q. (2007) An index to new species of Nematoda in Zootaxa from 2003 to 2006. Zootaxa, 1541, 65-68 (2 August).

Zhao, Z.Q. (2011) A review of the genus Trischistoma Cobb, 1913 (Nematoda: Enoplida), with descriptions of four new species from New Zealand. Zootaxa, 3045, 1-25 (29 September).

Zhao, Z.Q. \& Buckley, T.R. (2009) Phylogenetic analysis of nematode nuclear 18S rDNA sequences indicates the genus Tripylina Brzeski, 1963 (Nematoda: Tripylidae de Man, 1876) should be placed in Enoplida. Zootaxa, 2238, 25-32 (23 September).

Zhao, Z.Q., Li, D.M. \& Buckley, T.R. (2012) Analysis of primary structure loops from Hairpins 35 and 48 of the Nematoda SSU rRNA gene provides further evidence that the genera Tripylina Brzeski, 1963, Trischistoma Cobb, 1913 and Rhabdolaimus de Man, 1880 are members of Enoplida. Zootaxa, 3208, 41-57 (27 Feburary).

Zhuo, K., Cui, R.Q., Ye, W.M., Luo, M., Wang, H.H., Hu, X.N. \& Liao, J.L. (2010) Morphological and molecular characterization of Aphelenchoides fujianensis n. sp. (Nematoda: Aphelenchoididae) from Pinus massoniana in China. Zootaxa, 2509, 39-52 (17 June).

Zhuo, K., Liao, J.L., Cui. R.Q. \& Li, Y.Z. (2009) First record of female intersex in Hirschmanniella shamimi Ahmad, 1972 (Nematoda: Pratylenchidae), with a checklist of intersexes in plant nematodes. Zootaxa, 1973, 61-68 (9 June).

Zhuo, K., Wang, H.H. \& Liao, J.L. (2010) Neodolichodorus sinensis sp. nov. (Nematoda: Dolichodoridae) from China. Zootaxa, 2362, 63-68 (18 February).

Zullini, A. (2012) What is a nematode? Zootaxa, 3363, 63-64 (3 July). 